\title{
Der Anteil an Honigtau und die elektrische Leitfähigkeit des Honigs
}

\author{
H Pechhacker \\ Höhere Bundeslehr- und Versuchsanstalt für Wein- und Obstbau mit Institut \\ für Bienenkunde, Abteilung Bienenzüchtung, A-3293 Lunz am See, Österreich
}

(Eingegangen 1 Mai 1990; angenommen 15 Juni 1990)

\begin{abstract}
Zusammenfassung - An drei speziell vorbereiteten Völkern wurde während einer Honigtautracht der Honigtauanteil am Gesamtzuckereintrag durch die Analyse der Honigblaseninhalte heimkehrender Trachtbienen festgestellt. Der Honigtauanteil wurde mit folgenden Merkmalen des Honigs verglichen : Elektrische Leitfähigkeit, Anteil Honigtauelemente pro mg Zucker, Verhältnis Pollen nektarblütiger zu Pollen nektarloser Pflanzen pro mg Zucker und Pollen nektarblütig zu Honigtauelementen pro mg Zucker. Ein höherer Anteil an Honigtau am Gesamtzuckereintrag bewirkt im Honig der Versuchsvölker eine höhere elektrische Leitfähigkeit. Ein höherer Anteil an Honigtau hat eine geringere Anzahl an Pollen und Honigtauelementen pro mg Zucker zur Folge. Zwischen dem Verhältnis Anzahl Pollen nektarblütiger Pflanzen zu Pollen windblütiger Pflanzen bzw Anzahl Pollen nektarblütig zu Anzahl Honigtauelemente und dem Anteil an Honigtau besteht eine negative Korrelation.
\end{abstract}

Honig / elektrische Leittähigkeit / Honigtau / Honigtauelemente / Pollengehalt

\section{EINLEITUNG}

Orjewskij (1954) stellte fest, daß Honigtauhonige eine höhere elektrische Leitfähigkeit $(=\mathrm{EL}$ im weiteren Text) aufweisen als Blütenhonige. Vorwohl (1964a und 1964b) stellte ebenfalls eine Verbindung zwischen dem Anteil an Honigtau im Honig und der EL des Honigs fest. Die von inm (Vorwohl, 1964a) dargestellte, verbesserte Methodik gilt nach wie vor als gute Handhabe zur Feststellung des Honigtauanteiles im Honig. Dies wird durch die Arbeiten vieler anderer Autoren bestätigt.

Da die bisherigen Arbeiten nur auf der Schätzung des tatsächlichen Honig- tauanteiles im Honig beruhen (aufgrund der praktischen imkerlichen Beobachtung und Erfahrung), bestehen im Aussagewert der EL des Honigs noch gewisse Unsicherheiten. In der vorliegenden Arbeit stellten wir uns daher die Frage bezüglich des Zusammenhanges des möglichst exakt geschätzten Anteiles an Honigtau am gesamten Zuckereintrag für das Zustandekommen eines Honigs und dessen EL.

\section{METHODE}

Die Untersuchungen wurden 1987 zwischen dem 30 Juni und 21 Juli während einer ergiebigen Honigtautracht (Fichtentracht von 
Physokermes hemicryphus Dalman 1825/26, Cinara pilicornis Hartig 1841 und Cinara costata Zett, 1828) in Lunz am See durchgeführt. Der Durchschnittsertrag an Waldhonig bei den Leistungsprüfvölkern der umliegenden Stände betrug $25 \mathrm{~kg}$.

\section{Versuchsvölker}

Am 29 Juni wurden drei Bienenvölker auf Kunstschwärme abgekehrt und ohne jede Fütterung in der beginnenden Waldtracht auf Mittelwände gesetzt. Diese Völker hatten also keinerlei Vorräte an Honig, Pollen oder Brut.

\section{Probennahme}

Regelmäßig wurde zu verschiedenen Tageszeiten eines jeden Trachttages von jedem Volk getrennt eine Probe von rund 50 heimkehrenden Sammelbienen genommen. Wenn sich am vorübergehend geschlossenen Flugloch ausreichend Bienen gesammelt hatten, wurden diese in flüssigem Stickstoff abgetötet und bis zur Aufarbeitung bei $-18^{\circ} \mathrm{C}$ gelagert.

Am 21 Juli wurde die Entnahme von Bienenproben beendet und zugleich von jeder Wabe der drei Völker (insgesamt 26 Waben) eine Probe verdeckelten Honigs durch Herauspipettieren des Honigs aus den Zellen gezogen. Dieser Honig wurde bis zur Analyse bei $-18^{\circ} \mathrm{C}$ gelagert. Bei der Entnahme der Honigprobe aus der Wabe wurde besonders darauf geachtet, daß der Honig nur aus Zellen ohne Pollen herauspipettiert wurde, damit jede dadurch entstehende sekundäre Einstäubung mit Pollen vermieden wurde.

\section{Erhobene Daten von den Bienen}

- Honigblasengewicht in mg;

- Zucker mg je Honigblase (Biene); der Zuckergehalt wurde photometrisch nach der Anthron-Methode (Gesamtzuckerbestimmung; Humer, 1981) bestimmt. Die Gesamtanzahl der untersuchten Honigblasen und die dabei erfaßte Zuckermenge sind in der Tabelle I dargestellt;

- Vor der Gesamtzuckerbestimmung wurde von jedem Honigblaseninhalt die botanische Herkunft bestimmt. Anhand der Pollen bzw Honigtauelemente und der Konsistenz des Honigblaseninhaltes konnten Honigtau und Nektar auseinandergehalten werden. Honigblaseninhalte mit $9,27 \%$ des insgesamt untersuchten Zuckers konnten nicht eindeutig Honigtau oder Nektar zugeordnet werden (Tabelle 1). Sie wurden bei der Auswertung dem Nektar zugerechnet. Der prozentuelle Anteil des Honigtaues bzw Nektars am Gesamtzuckeraufkommen aus dieser Tracht konnte somit ziemlich genau pro Volk ermittelt werden. Der prozentuelle Anteil an Honigtau konnte nur jeweils für das gesamte Volk, nicht aber für jede einzelne Wabe getrennt erhoben werden, da die Verteilung des über die Honigblasenuntersuchung festgestellten Eintrages im Volk nicht kontrollierbar ist.

\section{Erhobene Daten an den Honigproben}

- Anzahl der Pollen (gesamt; nektarblütig; windblütig) pro mg Zucker;

- Anzahl der Honigtauelemente pro mg Zucker; hier wurde nicht auseinandergehalten, ob $\mathrm{zB}$ die gefundenen Pilzsporen tatsächlich vom Honigtau oder aber vom Nektar stammten. Besonders in Honigblaseninhalten mit Nektar von Cirsium (Pollen der Cirsium-Gruppe) wurden nämlich häufig neben Sporen von Kreuzhefen auch andere Pilzsporen gefunden;

- Die EL nach der von Vorwohl (1964a) angegebenen Methode : Messung in einer Lösung mit $20 \%$ Trockensubstanz bei $20^{\circ} \mathrm{C}$;

Diese Daten des Honigs wurden von jeder einzeinen Wabe erhoben. Aus den Ergebnissen der Proben der einzelnen Waben wurde für jedes Volk der jeweilige Durchschnittswert errechnet. Die Daten wurden mit herkömmlichen statistischen Verfahren ausgewertet.

\section{ERGEBNISSE}

Die Honige der Versuchsvölker entsprachen im sensorischen Test und in 
Tabelle I. Die Durchschnittswerte pro Volk und gesamt von Anteil Honigtau am Gesamtzuckereintrag, elektrische Leitfähigkeit des Honigs und Anzahl der Honigtauelemente, Pollen windblütiger, nektarloser und nektarblütiger Pflanzen pro mg Zucker.

\begin{tabular}{|c|c|c|c|c|}
\hline \multirow[t]{2}{*}{ Merkmal } & \multicolumn{3}{|c|}{ Durchschnittswerte } & \multirow{2}{*}{$\begin{array}{l}\text { Gesamt- } \\
\text { durchschnitt }\end{array}$} \\
\hline & Volk 43 & Volk 141 & Volk 198 & \\
\hline \multicolumn{5}{|l|}{$\%$ Honigtau am } \\
\hline Gesamtzuckereintrag & 56,74 & 70,46 & 68,50 & 65,23 \\
\hline $\begin{array}{l}\text { Elektrische Leit- } \\
\text { fähigkeit }(\mathrm{ms} / \mathrm{cm})\end{array}$ & 1,100 & 1,162 & 1,133 & $\begin{array}{c}1,134 \\
( \pm 0,0542)\end{array}$ \\
\hline $\begin{array}{l}n \text { Honigtauelemente/ } \\
\text { mg Zucker }\end{array}$ & 2,31 & 1,43 & 1,41 & $\begin{array}{r}1,79 \\
( \pm 1,53)\end{array}$ \\
\hline $\begin{array}{l}n \text { Pollen windblütig/ } \\
\text { mg Zucker }\end{array}$ & 0,35 & 0,18 & 0,16 & $\begin{array}{r}0,23 \\
( \pm 0,26)\end{array}$ \\
\hline $\begin{array}{l}n \text { Pollen nektarlos/ } \\
\text { mg Zucker }\end{array}$ & 0,76 & 0,75 & 0,16 & $\begin{array}{r}0,57 \\
( \pm 0,79)\end{array}$ \\
\hline $\begin{array}{l}n \text { Pollen nektar- } \\
\text { blütig/mg Zucker }\end{array}$ & 1,21 & 1,09 & 0,25 & $\begin{array}{r}0,86 \\
( \pm 0,84)\end{array}$ \\
\hline $\begin{array}{l}\text { nuntersuchte } \\
\text { Honigblasen }\end{array}$ & 452 & 478 & 453 & 1383 \\
\hline $\begin{array}{l}\text { In der Untersuchung } \\
\text { erfaßter Zucker mg }\end{array}$ & 7388 & 7855 & 8026 & 23269 \\
\hline $\begin{array}{l}\text { Nicht eindeutig } \\
\text { Honigtau oder Nektar } \\
\text { (\% von erfaßten Zucker) }\end{array}$ & 10,1 & 9,0 & 8,7 & 9,27 \\
\hline $\begin{array}{l}n \text { untersuchte Honig- } \\
\text { proben (Waben) }\end{array}$ & 8 & 8 & 10 & 26 \\
\hline
\end{tabular}

ihren sonstigen Eigenschaften dem Standard typischer Fichtenhonige aus der Region. Der durchschnittliche Wassergehalt der Honigproben betrug 16,2\%. Bei den Völkern 141 und 198 war der Honig zu einem geringen Ausmaß (rund 10-15\%) bereits in der Zelle auskristallisiert (Melezitosehonig). Speziell bei diesen Völkern war der Anteil an "Honigtau kristallisiert" mit $26 \%$ am
Gesamtzuckereintrag am höchsten. "Honigtau kristallisiert" bedeutet, daß der Honigblaseninhalt bei der Tiefkühllagerung der Bienenproben schon nach einer Woche in der Honigblase auskristallisiert war, während der Honigblaseninhalt bei "Honigtau flüssig" noch nach zwei Jahren Gefrierlagerung flüssig war. Dieser "Honigtau kristallisiert" wies im Durchschnitt $41,12( \pm 2,19) \%$ Melezitose 
am Gesamtzucker auf (Pechhacker, 1990).

In der Tabelle I sind die Durchschnittswerte der einzelnen Völker dargestellt. In den Abbildungen 1 und 2 werden die signifikanten Zusammenhänge zwischen dem prozentuellen Honigtauanteil am Honig und den verschiedenen Kriterien der Honige dargestellt. Hier konnten zur statistischen Auswertung jeweils nur die Durchschnittswerte herangezogen werden (dies entspricht nur jeweils drei Wertepaaren).

Bei den Ergebnissen aus den einzelnen Waben $(n=26)$ konnte nur in den Beziehungen EL zu Anzahl Honigtauelemente pro $\mathrm{mg}$ Zucker $(r=-0,40 ; P<$ $0,05)$ und $E L$ zu Verhältnis Pollen nektarblütig zu Anzahl Honigtauelemente $(r=-0,38 ; P<0,05)$ ein signifikanter Zusammenhang gefunden werden. Die anderen Beziehungen ließen wegen der großen Streuung zwischen den Waben innerhalb eines Volkes nur Trends erkennen.

\section{DISKUSSION}

Der gefundene signifikante Zusammenhang zwischen dem prozentuellen Anteil an Honigtau am Gesamtzuckereintrag und der durchschnittlichen EL (Abb 1A) bestätigt die bisherigen Annahmen (Orjewskij, 1954; Vorwohl, 1964a und b; Dustmann, 1967; Bianchi, 1980) sehr klar. Anhand der EL kann daher der Anteil an Honigtau am Zustandekommen des Honigs und damit die botanische Herkunft gut erkannt werden. Allerdings müssen weitere Untersuchungen an den verschiedenen Honigtautrachten bzw Regionen durchgeführt werden, um generelle Standards zu schaffen.

Nicht den Erwartungen entsprach die Beziehung EL zu Anzahl der
Honigtauelemente pro mg Zucker. Auch bei den anderen Sedimentbestandteilen (Pollen nektarblütig, Pollen windblütig bzw nektarlos) zeigte sich eine Tendenz, daß bei höherem Anteil an Honigtau die Anzahl der Sedimentbestandteile pro mg Zucker generell niedriger ist. Diese Ergebnisse wurden durch die in $A b b 2 A$ und $B$ dargestellten Beziehungen zwischen dem Anteil an Honigtau und der Anzahl Pollen windblütig pro $\mathrm{mg}$ Zucker bzw EL zu Anzahl Honigtauelemente pro mg Zucker (Abb 1B) sowohl beim Durchschnitt der einzelnen Völker als auch bei den einzelnen Waben bestätigt. Als
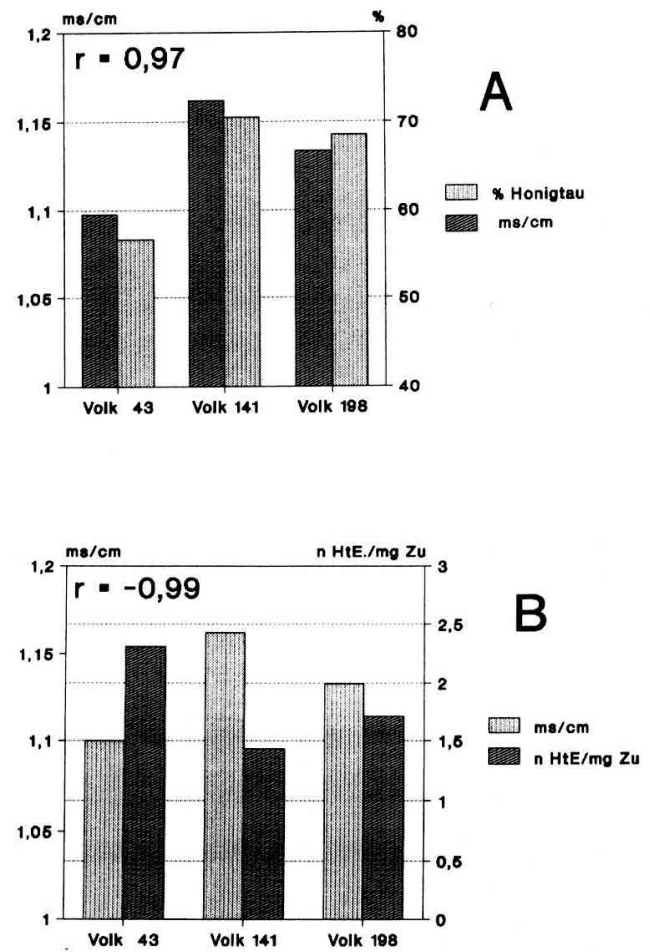

Abb 1. Zusammenhang zwischen dem Anteil an Honigtau am Gesamtzuckereintrag und der EL. (A) bzw dem Anteil an Honigtauelementen pro mg Zucker im Honig (n HtE/mg Zucker) und der EL (B). 
Begründung für diese Beziehung könnte ein generell geringerer Anteil an Sedimentbestandteilen pro $\mathrm{mg}$ eingetragenem Zucker im Honigtau angenommen werden. Beweise hierzu fehlen allerdings.

Die signifikanten Beziehungen zwischen dem Anteil an Honigtau am Gesamt-
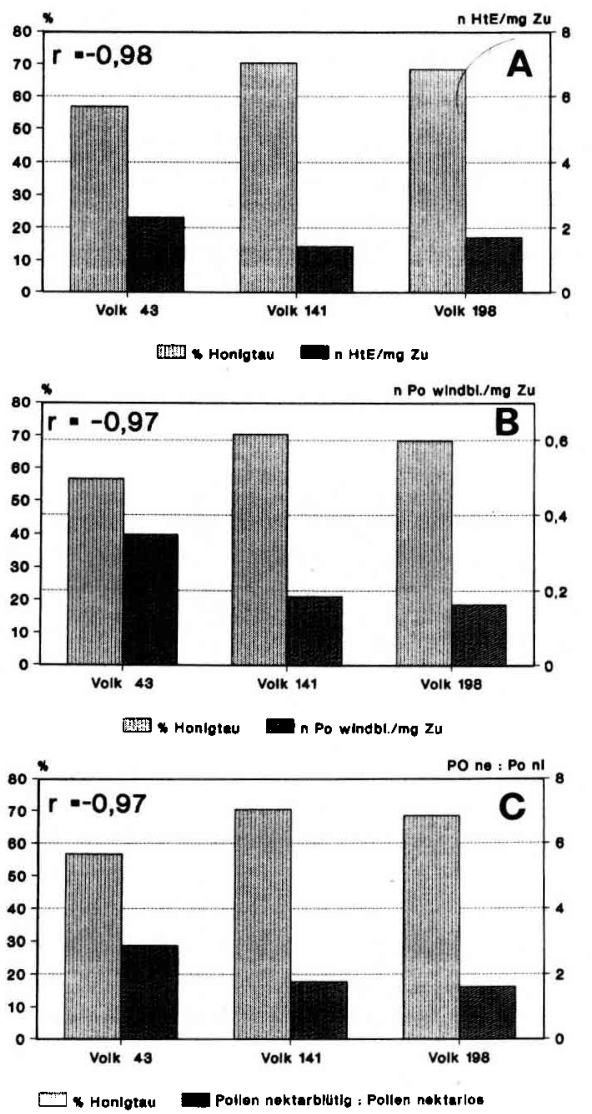

Abb 2. Zusammenhang zwischen dem Anteil an Honigtau am Gesamtzuckereintrag (\%) und der Anzahl Honigtauelemente pro $\mathrm{mg}$ Zucker im Honig (n HtE/mg Zucker; A), der Anzahl Pollen windblütiger Pflanzen pro $\mathrm{mg}$ Zucker (n Po windbl/mg Zucker; B) und dem Verhältnis Pollen nektarblütger zu Pollen nektarloser Pflanzen (Po ne : Po nl; C). zuckereintrag und dem Verhältnis Pollen nektarblütig zu Pollen nektarlos (Abb 2C) und der Beziehung EL zu Verhältnis Pollen nektarblütig zu Honigtauelemente, bestätigen die Richtigkeit der bisher geübten Praxis in der Honig-Pollen-Analyse : honigtaureicherer Honig hat im Bezug auf die Anzahl der Pollen nektarblütig mehr Pollen nektarlos bzw mehr Honigtauelemente im Sediment.

\section{Summary - Honeydew content and electrical conductivity of honey. During} the honey flow from honeydew in Lunz am See, Austria, between June 30th and July 21st 1987, samples of returning fieldbees from 3 specially prepared colonies (without any reserve of honey and pollen) were collected daily at various times. The bee samples were killed in liquid nitrogen and stored at $-18^{\circ} \mathrm{C}$. At the end of the experiment honey samples were also taken from each comb of the colonies.

The following data were collected from the bees: weight of the honeysac, sugar content in the honeysac (Anthron method = total sugar analysis) and the botanical origin of the honeysac content (on the basis of pollen grains and fungus spores). Measurements of the honey samples were as follows : electrical conductivity, number of pollen grains (nectar plants and nectarless plants) and honeydew components (fungus spores) in the honey sediment per mg sugar.

Significant correlations were found between electric conductivity and sugar content originating from honeydew and number of honeydew components per $\mathrm{mg}$ sugar in the honey (fig 1A, B).

Significant negative correlations were also found between the percentage of honeydew sugar in the honey and the number of honeydew components in the honey sediment per mg sugar, the number of pollen grains from wind-pollinating plants per $\mathrm{mg}$ sugar and the relation of pollen grains 
of nectar plants to pollen of nectarless plants per mg sugar (fig $2 \mathrm{~A}, \mathrm{~B}, \mathrm{C}$ ). The result of higher honeydew content in the honey is a significant higher electric conductivity, a lower relation between the number of pollen grains from nectarplants to pollen of nectarless plants per mg sugar and a general lower content of sediment components per mg sugar in the honey.

\section{honey / electrical conductivity / honey- dew / honeydew component / pollen content}

Résumé - Teneur en miellat et conductibilité électrique du miel. Durant la miellée de miellat à Lunz am See (Autriche) du 30 juin au 21 juillet 1987, on a prélevé, chaque jour à des heures variables, des échantillons de butineuses rentrant à la ruche et appartenant à 3 colonies expérimentales dépourvues de réserves de miel et de pollen. Les abeilles capturées ont été tuées dans l'azote liquide et conservées à $-18^{\circ} \mathrm{C}$. A la fin de l'expérience, des échantillons de miel ont également été prélevés dans chacun des rayons des colonies.

Les données suivantes ont été notées :

- pour le jabot des abeilles : poids, teneur en sucres (méthode Anthrone = analyse des sucres totaux) et origine botanique (d'après les grains de pollen et les spores de champignons);

- pour le miel : conductibilité électrique, nombre de grains de pollen (plantes nectarifères et plantes non nectarifères) et d'indicateurs de miellat (spores de champignons) par mg de sucre.

Des corrélations significatives ont été trouvées entre la conductibilité électrique, la teneur en sucre provenant du miellat et les indicateurs de miellat (spores de champignons) par $\mathrm{mg}$ de sucre dans le miel (figure $1 A$ et $B$ ). Des corrélations significativement négatives ont été aussi trouvées entre les pourcentages de sucre de miellat dans le miel et le nombre d'indi- cateurs de miellat dans le sédiment du miel par mg de sucre, le nombre de grains de pollen provenant de plantes anémophiles par mg de sucre et le rapport grains de pollen de plantes nectarifères / grains de pollen de plantes non nectarifères par $\mathrm{mg}$ de sucre (figure $2 \mathrm{~A}, \mathrm{~B}$ et $\mathrm{C}$ ). La conséquence d'une teneur en miellat plus élevée est une conductibilité électrique du miel significativement plus élevée, un rapport plus faible grains de pollen de plantes nectarifères / grains de pollen de plantes non nectarifères par $\mathrm{mg}$ de sucre et une teneur générale plus faible en éléments de sédiment par mg de sucre dans le miel.

miel / conductibilité électrique / miellat / indicateur de miellat / teneur en pollen

\section{LITERATUR}

Bianchi EM (1980) Die elektrische Leitfähigkeit in Honigen aus Santiago des Estero, Argentinien. Apidologie 11, 25-28

Dustmann JH (1967) Messungen von Wasserstoffperoxid und Enzymaktivität in mitteleuropäischen Honigen. $Z$ Bienenforsch 9, 66-73

Humer J (1981) Nektar- und Pollenuntersuchungen an sammelnden Honigbienen. Diplomarbeit, Botanisches Institut der Universität für Bodenkultur, Wien

Orjewskij TD (1954) Elektrolytische Methode zur Entdeckung des Honigtaues. Pchelovodstvo (9), 42 (in Russisch)

Pechhacker H, Praznik W, Klaus J (1990) Untersuchungen über das Zuckerspektrum in Honigblaseninhalt und Honig. Apidologie 21, 447-455

Vorwohl G (1964a) Die Messung der elektrischen Leitfähigkeit des Honigs und die Verwendung der Meßwerte zur Sortendiagnose und zum Nachweis von Verfälschungen mit Zuckerfütterungshonigen. $Z$ Bienenforsch 7, 37-47

Vorwohl G (1964b) Die Beziehungen zwischen der elektrischen Leitfähigkeit der Honige und ihrer trachtmäßigen Herkunft. Ann Abeille 7 (4), 301-309 